\title{
Social Dynamics of Expectations and Expertise: AI in Digital Humanitarian Innovation
}

\author{
GUILLAUME DANDURAND \\ UNIVERSITÉ DE SHERBROOKE \\ François ClaveaU ${ }^{2}$ \\ UNIVERSITÉ DE SHERBROOKE \\ JEAN-FRANÇOIS DUBÉ ${ }^{3}$ \\ UNIVERSITÉ DE SHERBROOKE \\ FLORENCE MiLLERAND \\ UNIVERSITÉ DU QUÉBEC À MONTRÉAL
}

\begin{abstract}
Public discourse typically blurs the boundary between what artificial intelligence (AI) actually achieves and what it could accomplish in the future. The sociology of expectations teaches us that such elisions play a performative role: they encourage heterogeneous actors to partake, at various levels, in innovation activities. This article explores how optimistic expectations for AI concretely motivate and mobilize actors, how much heterogeneity hides behind the seeming congruence of optimistic visions, and how the expected technological future is in fact difficult to enact as planned. Our main theoretical contribution is to examine the role of heterogeneous expertises in shaping the social dynamics of expectations, thereby connecting the sociology of expectations with the study of expertise and experience. In our case study of a humanitarian organization, we deploy this theoretical contribution to illustrate how heterogeneous specialists negotiate the realization of contending visions of "digital humanitarianism."
\end{abstract}

\section{Keywords}

expectations; expertise; artificial intelligence; digital humanitarianism; performativity

Guillaume Dandurand, Email: guillaume.dandurand@usherbrooke.ca

2François Claveau, Email: francois.claveau@usherbrooke.ca

Jjean-François Dubé, Email: jean-francois.dube@usherbrooke.ca

"Florence Millerand, Email: millerand.florence@uqam.ca

Copyright (C) 2020 (Guillaume Dandurand, François Claveau, Jean-François Dubé, Florence Millerand). Licensed under the Creative Commons Attribution Non-commercial No Derivatives (by-nc-nd). Available at estsjournal.org. 


\section{Introduction}

In representations of artificial intelligence (AI), the distinction between what AI could accomplish in the future and how technologies that fall under the umbrella of AI currently perform is often obfuscated. In 2016 for instance, the car company Tesla advertised its Model S as a smart car that would function "without human input by the end of 2017" (Stilgoe 2018, 25-26). Just a few years before that, IBM envisioned deploying its AI Watson Technology in the health care sector as "an evidence-based clinical decision support system" (Ferrucci et al. 2013, 93) to assist physicians in making diagnoses. Today, none of these plans have yielded convincing results. In contrast, other AI-based innovations have delivered on their promises. For instance, Google uses AI to suggest quick email replies based on the content of incoming messages. Such successes do exist; however, representations of future applications of AI are too often presented in public discourse as inevitable outcomes of technological development rather than challenging processes that may fail to meet initial expectations. Expectations of $\mathrm{AI}$ and the realization of those expectations are conflated, to the extent that some people are unadvisedly heralding this moment in history as the advent of the fourth industrial revolution (Schwab 2017).

In this article, we take a more cautious approach, while focusing on the analytical gap between technological expectations for $\mathrm{AI}$ and the challenging innovation processes fueled by these expectations. We turn to science and technology studies (STS), and more specifically to the cohesive body of work known as the sociology of expectations (Borup et al. 2006; Konrad et al. 2017), to probe how promissory discourses on AI shape innovation processes. Our theoretical contribution is to enrich the framework of the sociology of expectations with key insights from Harry Collins and Robert Evans' research program, "Studies of Expertise and Experience" (2002, 2007). This synthesis allows us to analyze the role that heterogeneous expertises ${ }^{6}$ play in influencing the social dynamics of expectations. At the micro-level, we argue, integrating these two STS literatures offers an insightful perspective on how actors, informed by differing expertises, translate generic collective expectations into variegated actor-specific expectations as they engage in innovation processes.

We use this theoretical framework to analyze a case study of AI-based innovation by a small unit of a transnational humanitarian organization (hereafter, "THO"). Humanitarianism is by nature reactive: in times of crisis, humanitarian workers react to natural or human-made

${ }^{5}$ The term "innovation" was used extensively by the interlocutors we interviewed as part of our research on digital humanitarianism. For them, innovation refers to two characteristics of their activities: the use of recent technology and the quest to change practices in the humanitarian sector. In this article, we use the term innovation the same way as these interlocutors have, instead of as an analytic category (see Godin and Vinck 2017).

"Although "expertise" is typically used in the singular form, we follow Collins and Evans (2002; 2007) in pluralizing it to highlight how varied it is.

-To preserve the anonymity of the interlocutors that we interviewed, we use gender-neutral pseudonyms to refer to them throughout this article. We also anonymize the name of the organization for which they work and the name of the digital humanitarian project. The acronym VirtEYE, which we introduce later in the paper, is fictitious. 
disasters to provide assistance to people in dire need of aid-to "save lives" as the maxim goes. For some humanitarian workers, the design and implementation of digital technologies will render humanitarian responses more efficient. Indeed, this opinion captured the generic expectations regarding AI-based innovations in the humanitarian sector when the small unit of humanitarians initiated their project: AI was widely (and vaguely) believed to be an important way to improve the efficiency of humanitarian reactions in order to save even more lives. This promissory vision is known as "digital humanitarianism" (Meier 2012, 2015). In public discourse, this vision is associated with a prominent researcher and advocate for digital humanitarianism, Patrick Meier, who claims that "anyone can be a digital humanitarian, absolutely no experience necessary; all you need is a big heart and access to the Internet" $(2015,1)$. Such a vision drives organizations such as THO to develop complex digital instruments for humanitarian purposes. Existing research on digital humanitarianism has already critiqued this type of optimistic view of technology in humanitarian contexts (Burns 2014; 2015; Crawford and Finn 2015; Duffield 2016; Roth and Luczak-Roesch 2020). In this line, our article scrutinizes innovation processes through which optimistic visions of AI are mediated by heterogeneous expertise to form divergent actorspecific expectations of technology.

The next section presents our theoretical framework, followed by the methods section. Then, we use the evidence collected during our fieldwork to analyze how expertise and experience shape the social dynamics of expectations and, in turn, the multifaceted challenges of innovation processes during the development of digital humanitarian technologies.

\section{The Sociology of Expectations and the Heterogeneity of Expertise}

The word "expectation" descends from the Latin exspectatio, which conveys a future-oriented state of "looking for" or "waiting for" (Borup et al. 2006). Since the 1990s, STS scholarship has produced a rich and cohesive literature on the sociology of expectations by probing how representations of future technologies participate in innovation processes. ${ }^{.}$In this article, we use the well-established definition of Mads Borup and colleagues: "technological expectations [are] real-time representations of future technological situations and capabilities" (Borup et al. 2006, 286). This section describes our theoretical framework, which combines seminal elements from the "Studies of Expertise and Experience" research program (Collins and Evans 2002, 2007) with the sociology of expectations. We contend that this theoretical synthesis is helpful for understanding the dynamics that emerge between collective expectations and actor-specific expectations at the micro-level of innovation processes. In the next section, our case study exemplifies the usefulness of this theoretical contribution.

Research on the social dynamics of technological expectations has coalesced around a number of general propositions about these expectations (cf. Borup et al. 2006; Konrad et al. 2017). First, technological expectations are value-laden. They are not neutral descriptions of what is likely to happen, because in focusing on some positive or negative characteristics of what is

"Harro van Lente's (1993) PhD thesis constituted a watershed moment in the origination of this literature. 
deemed likely, these expectations suggest the desirability or undesirability of a technological path. Expectations have a positive valence when they evoke hopes and optimism and a negative valence when they educe fears and pessimism (Mulkay 1993).

Second, technological expectations range from the extremely generic representation of a world with "more advanced technology" to very narrow representations of specific technological changes (e.g., a new version of an operating system). In the literature on this topic, investigators often refer to generic representations of what the technological world could be as "visions" (Konrad et al. 2017, 467) or "sociotechnical imaginaries" (Jasanoff and Kim 2009, 119). Note here how "visions" and "imaginaries" take the plural form. They do so because heterogeneous generic representations of a technological future coexist in society, from blind faith in technological progress to dystopian fears.

Third, expectations of some specific technologies follow a typical temporal pattern (Brown and Michael 2003; Dedehayir and Steinert 2016; Geels and Smit 2000; van Lente et al. 2013). Some technological developments generate a rapid inflation of enthusiasm. In this initial phase, projections of what the technology could accomplish must be forecasted from limited concrete results. After a while, when the initial inflated expectations are not realized, enthusiasm for the technology plummets. There is a growth in feelings of disillusionment, followed by a recalibration of expectations, during which resources are either diverted away from the technology or redirected such that innovation processes lead to more modest or different applications. This temporal pattern is so typical of innovation processes that it has generated a famous management tool: the "hype cycle" that Gartner Inc. sells to businesspersons around the world, with the objective of "mastering the hype cycle" (Fenn and Raskino 2008).

Fourth, expectations are performative (Borup et al. 2006). Although the present often turns out to be different from what was expected, expectations are far from being superfluous representations that are superimposed on autonomous technological development. Rather, the valence and the intensity of expectations act on those technological developments. Strongly negative expectations kill technological exploration, while overly optimistic expectations propel whole industries. Unsurprisingly, expectation-building-or hype-is central to the strategic considerations of technological innovators, who formulate explicit promises in an attempt to make more likely the successes that they predict (Brown and Michael 2003; Joly 2015; van Lente 1993). Through such promises and other discursive activities, expectations guide innovations, coordinate agendas, and encourage and legitimize financial and human engagements (van Lente and Rip 1998).

Fifth, expectations are maintained and modified through the relation between actorspecific and collective expectations (Konrad 2006). Actor-specific expectations are held or attributable to certain actors or groups of actors. For instance, some humanitarian workers expect that current investment in machine learning by their own humanitarian organizations would be a

'The hype cycle model that Gartner Inc. sells to clients who hope to predict the state of the technological future (Fenn and Raskino 2008) is fraught with incongruences, inconsistencies, and ambiguities (Dedehayir and Steinert 2016; Mullany 2016). 
waste of scarce and much-needed resources. In contrast, collective expectations are "the expectations of generalised others" (Konrad 2006, 433). The same humanitarian workers readily admit that, by and large, their colleagues expect much from future applications of machine learning, although these workers distance themselves from this hype. As Figure 1 from Konrad $(2006,432)$ illustrates, actors-partly based on their own expectations and their understandings of collective expectations-engage in discursive and innovative activities that, by being observed by others, have the potential to shape collective expectations. In turn, these collective expectations may be internalized by heterogeneous actors, thereby shaping their individual expectations.

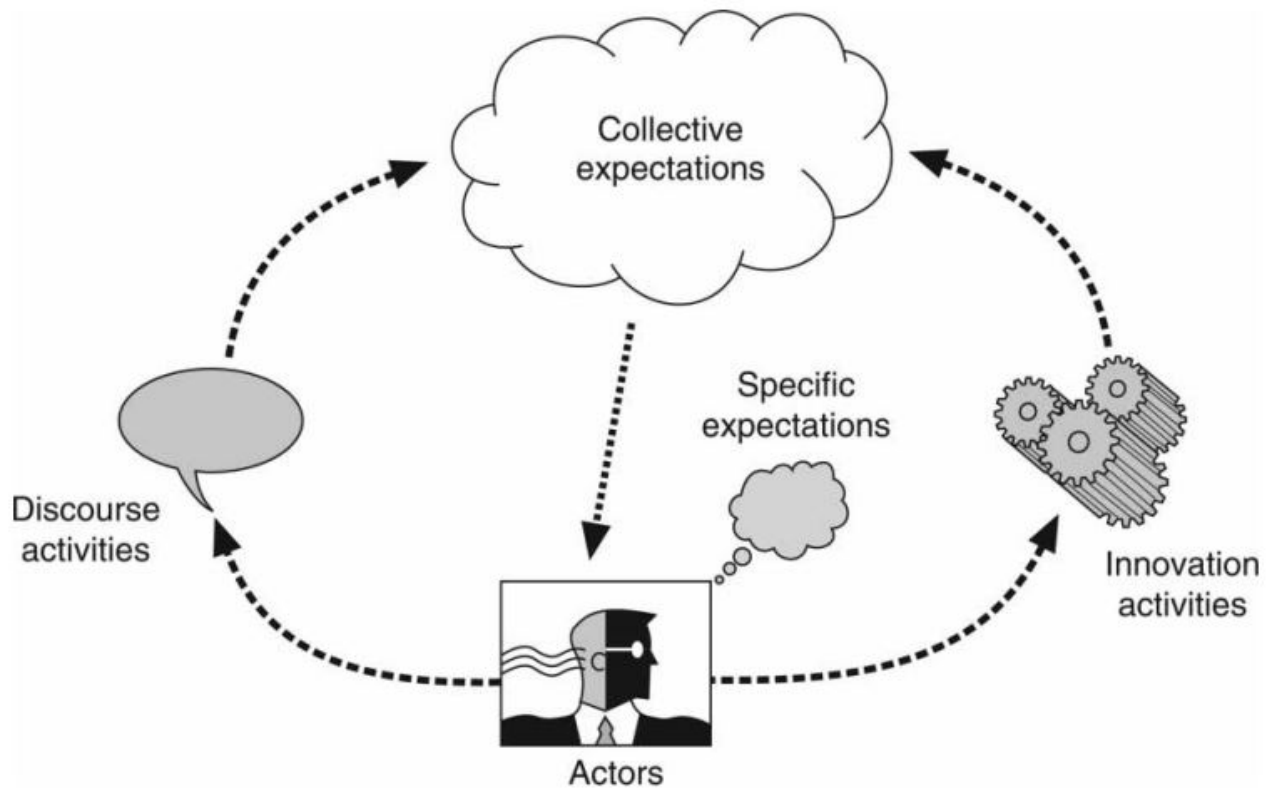

Figure 1: Konrad's $(2006,432)$ schematic representation of the relationship between actors' activities and collective expectations

Finally, the content of actor-specific expectations is heterogeneously distributed across social groups (Borup et al. 2006, 292). Obviously, actors at the periphery of research and technological innovation tend to have a much less complex understanding of new technology, as compared with those at the center. For instance, few persons outside of the machine-learning community can describe in detail what a convolutional neural network is. However, having a more detailed understanding of a technology does not ensure that technological specialists formulate more accurate expectations about how the technology will change various social domains, in part because they typically lack relevant expertise in the domains of application. ${ }^{10}$

\footnotetext{
10 We already alluded to another reason why technological specialists typically express untrustworthy expectations about the deployment of their technology: for the strategic purpose of building positive
} 
This raises the question that serves as an entry point for our contribution to the sociology of expectations: how does expertise shape one's specific expectations? We turn to the work of Collins and Evans in their research program, "Studies of Expertise and Experience" $(2002,2007)$. Collins and Evans have developed a topology of expertise that enables us to better understand how expertise influences the formulation of specific expectations among heterogeneous actors. According to them, expertise is a fluency in the "ways of going on and thinking" of a community or domain (Collins 2018, 68). This fluency is acquired tacitly through immersion in a relevant community. This conception of expertise distances itself from an attribution of expertise to only some social groups-e.g., scientists and other technical specialists. In fact, many kinds of expertise are not possessed by scientists or technological innovators, but rather by other social groups. For instance, humanitarian workers have a specialist expertise that engineers in machine learning lack because the latter are not immersed in the humanitarian domain."

This heterogeneous distribution of specialist expertises in society is the first theoretical component we take from Collins and Evans' work, since it closely relates to the heterogeneous distribution of expectations about a technology. The heterogeneity of expectations does not arise from a distinction between those who know versus those who do not know. Rather, it partly emerges through a distinction between those who are more fluent with the intricacies of the technology itself (one specialist expertise) versus those who are more fluent with other things, such as the possible contexts of application of the technology (other specialists expertises). This distinction is key for illuminating the social interactions that propel the dynamics of expectations (Konrad 2006). For instance, technological specialists tend to underestimate the challenges of adapting a technology that shows promising performance in a controlled environment to specific contexts (Geels and Smit 2000).

The other theoretical component that we borrow from Collins and Evans is the distinction between contributory and interactional expertises. This distinction is useful and cardinal to our argument as it provides new insights into the social dynamics of expectations, especially at the micro-level where a small group of heterogeneous specialists must work together towards the realization of techno-scientific projects. Contributory expertise refers to the capacity to contribute to the practices of a domain. On the other hand, interactional expertise is "the ability to master the language of a specialist domain in the absence of practical competence" (Collins and Evans 2007, 14). Acquiring interactional expertise requires some immersion in the domain, including direct interaction with its contributory experts. It enables one to engage with others who share fluency in the activities of a domain, but it does not require as much proficiency as contributing significantly to these activities would (Collins and Evans 2002). Most importantly, it is easier for contributory experts in one domain-in our case study, the two main domains are

collective expectations. See Frank W. Geels and Wim A. Smit (2000) for a discussion of failed technological expectations.

"Note that Collins and Evans' topology also includes "ubiquitous expertises," i.e., a fluency shared by almost everyone in a society-e.g., speaking the official language(s) of a country. We keep this category in the background in this article because what interests us is the encounter of heterogeneous specialist expertises. 
humanitarianism and computer programming - to acquire an interactional expertise in another domain than it is for them to acquire a second, full-fledged, contributory expertise. Although a diversity of contributory expertise may be critically important for the realization of a given technological project, we show in the next section that interactional expertises shape how actors navigate the coordination of heterogeneous specific expectations and the challenges of innovation processes. In sum, we contend that Collins and Evans' theoretical framework is helpful for examining: (1) how a topology of expertises shapes the formulation of actor-specific expectations; and (2) how the heterogeneity of expertise accounts for the dynamics of expectations at the micro-level, where the challenges of coordinating expectations appear to be inversely related to the level of interactional expertises among actors. It is these two elements from Collins and Evans's work on expertise that enrich the analysis of the social dynamics of expectations in our case study.

\section{Method}

Our article builds on 18 months of fieldwork conducted with THO between September 2018 and May 2020 as part of a collaborative research project on the construction of an AI-powered geographic information system (GIS). Collaborative research is an academic practice that mediates scholarly and practitioner knowledge (Vinatier and Morrissette 2015)." We drew on our multidisciplinary areas of social science expertise to provide assistance in the development of a digital humanitarian project, which we will refer to as Virtual Eye (VirtEYE). In turn, our research partners at THO offered us the opportunity to conduct participant-observation research on the development of VirtEYE in their humanitarian context. During our fieldwork, we formally conducted 11 interviews with THO workers (4 semi-directed and 7 directed interviews) and attended several meetings for the coordination of VirtEYE. The interviews and meetings were all organized by our THO research partners. These interviews and meetings were conducted online and lasted from 30 to 75 minutes each. Our THO partners also gave us access to the documentation accumulated throughout the innovation processes of VirtEYE, including prior and current budgets, funding applications, timelines, questionnaires, and other documents describing the GIS development.

While our data collection techniques relate to the participant-observation method, we use case study as a methodology. For Robert K. Yin, a case study research strategy is an empirical inquiry that "investigates a contemporary phenomenon within its real-life context," with no clear demarcating line between context and phenomenon (Yin 2003, 13). This research strategy must be adapted to a situation where data points are scarce in comparison to the number of potentially relevant variables. The adaptations involve, first, the use of multiple sources of evidence to allow

${ }_{12}$ Our participation in this collaborative research project has been informed by our critical engagement in the design and implementation of a digital humanitarian project, one in which we study the "practices of information technology design while embedded at a site of use, influencing production but without contributing to a 'bottom line'" (Vertesi et al. 2017, 172). 
for converging data and, second, "the prior development of theoretical propositions to guide data collection and analysis" (Yin 2003, 13-14).

In the next section, we use our theoretical framework combining the core propositions of the sociology of expectations with the concepts of contributory and interactional expertises to structure our analysis of the digital humanitarian project VirtEYE.

\section{Dynamics of Expectations of AI in Digital Humanitarianism: A Case Study}

As emphasized in the theoretical section, expectations are performative: they create momentum for the realization of techno-scientific visions through a series of innovation processes. At the micro-level, positive collective expectations help to motivate and guide actors to partake in these innovation processes. In what follows, we examine how the heterogeneous expertises of actors are central to the interpretation of collective expectations, the formulation of specific promises, and ultimately, the realization of a digital humanitarian vision.

\section{Initial Phase: Situating Actors and their Expertise}

In 2014, a group of specialists developed what Meier calls a "live crisis map" (2012, 89): an online GIS that provides real-time geo-localized information on the impacts of monsoon flooding in Jakarta, Indonesia. A GIS is a visual representation of the world that displays various sources and forms of information spatially on a map. ${ }^{33}$ This particular GIS serves as a digital platform on which users can input, store, retrieve, and exchange knowledge in real-time in order to improve the management of information during floods in Jakarta. The GIS uses social media to reach the local population and invite them to upload their first-hand observations of the impact of flooding on various urban infrastructures. Thus, the system crowd sources actionable information for governmental authorities, organizations, and other members of the population in times of crisis. In the words of two project members:

[The system] aims to research and develop open source software (OSS) for the integrated management of social media and API [application programming interface]-sourced data in order to make risk information open, accessible, and actionable by residents, government agencies, NGOs [non-governmental organizations], and private sector developers. (Holderness and Turpin 2015, 3)

When the pilot project was finally launched in late 2014, the system became an early digital humanitarian tool that harnesses crowdsourcing in order to enhance information management in times of natural disaster.

In 2017, two developers who played a key role in the development of this GIS met with experienced humanitarian workers from THO to develop a similar digital humanitarian project.

13GIS have existed since the 1960s and have evolved with technological developments (Mark et al. 1997). 
These developers brought with them a diverse set of expertise: they had contributory expertise in programming GIS and chatbots, as well as some degree of interactional expertise in humanitarian workflows. Even though they had never been on humanitarian missions themselves, they had developed a fair amount of interactive ability to translate the needs and constraints of humanitarian workers into digital solutions. These developers (hereafter, "the tech specialists") possessed a solid grasp of AI and its practical applications. However, the tech specialists had no prior experience implementing these sorts of machine-learning algorithms for humanitarian partners in the specific context of THO.

The workers from THO were at different stages of their careers in the humanitarian sector. Some had multiple decades of experience on humanitarian missions, while others were fresh out of graduate school and working on their first contract with THO. Taken together, these workers had considerable contributory expertise in humanitarian action, especially with respect to THO's organizational culture. They also held administrative positions and thus had well-tried interactive abilities to discuss the ins and outs of humanitarian work with outsiders. However, the interactional expertise of these workers (hereafter, "the humanitarian specialists") with regard to the development of digital technologies was modest.

In what follows, we describe the social dynamics of expectations to analyze the interactions of the tech and humanitarian specialists as they worked together to realize the same technoscientific future: an AI-powered GIS that is capable of supporting information management for THO. We focus on the professional position of these actors, and pay particular attention to their degree of contributory and interactional expertises as they engage with one another in the transition from collective expectations about automation to the formulation of specific expectations about AI in various phases of the innovation processes. Using Collins and Evans' research program on "Studies of Expertise and Experience" (2002; 2007) leads us to argue that actors involved in innovation processes do so on the basis of their own contributory and interactional expertises which, in turn, shape the social dynamics of expectations in particular ways. Following Mads Borup and colleagues (2006), we divide our case study according to typical phases of innovation processes.

\section{Phase 1: The Generative Force of Expectations}

In March 2017, the tech and humanitarian specialists partnered to co-develop a web-based GIS called VirtEYE. According to the first project charter of VirtEYE (2017), one significant challenge for humanitarianism consists in sourcing accurate and actionable information and sharing it among humanitarian workers in a timely manner. Charlie, one of the humanitarian specialists, emphasized that information management in the midst of a crisis can quickly become chaotic. In their decision to team up with the tech specialists, the humanitarian specialists were motivated by the most basic expectation that a GIS that visually displays much-needed information in times of crisis would "make our lives much easier!" (Charlie, online group meeting, 2019).

VirtEYE was inspired by the project taking place in Jakarta, but it departed from this earlier GIS project in significant ways, both in terms of what the system was meant to do and how 
it was meant to do it. Like the earlier system, VirtEYE shares geo-localized information between different actors-e.g., identifying where health infrastructures are located and which roads are functional to allow efficient response. However, the earlier system was deployed to share actionable information among local governments, NGOs, and city residents; in contrast, one of the core objectives of VirtEYE was to connect primarily humanitarian workers who are remotely located from one another. Furthermore, VirtEYE was developed to store data and records compiled during previous THO missions, in order to ease access to information that may be relevant for subsequent humanitarian interventions-e.g., the location of hospitals, helipads, and contact persons in a particular region. Knowledge of this sort constitutes the "institutional memory" of the organization-i.e., the vast amount of files, maps, and other archives produced in prior missions. Mining the institutional memory of the organization for relevant information in times of crisis has historically been time consuming. One of the derivative objectives of VirtEYE was thus to improve the storage and retrieval of this knowledge.

Even for an experienced humanitarian specialist, finding relevant and actionable information in a timely manner from past and current events is a challenging task-particularly given the lack of a common, homogeneous, and standard archival system across THO; disparities in logging logistical information across time and space; and frequent employee and volunteer turnover. Thus, the humanitarian specialists found hope in the collective expectations that AI would help with information management. At the time, optimistic techno-scientific visions about automation had gained prominence in several domains. Recent AI successes had fueled projections of a rapid increase in the permeation of automation in human lives. AI AlphaGo had beaten Lee Sedol, a legendary player in the world of Go, in March 2016. Media reports stated it was "a victory experts had predicted was still years away" (Borowiec 2016) and had interpreted the event as a sign that AI was improving more quickly than expected. At the end of 2016, Spectrum announced that 2017 was going to be "The Year of Self-Driving Cars and Trucks" (Hassler 2016). This over-enthusiasm for future applications of AI had reached the humanitarian sector as well. In 2018, a column published in the online newspaper Le Temps, shared with us by one of the humanitarian specialists, stated that the entire humanitarian sector would be transformed by AI." Unsurprisingly, given this state of collective expectations of AI, the tech and humanitarian specialists believed that VirtEYE was bound to be an AI-powered digital humanitarian tool, the first of its kind in THO (Project Charter 2017). In a nutshell, both the tech and humanitarian specialists held a similar techno-scientific vision of digital humanitarianism: automation and AI technologies were expected to be technological triggers for the enhancement of information management in times of crisis.

During the first six months of the project, the team surveyed 52 humanitarian workers and analyzed their answers. Based on the needs shared by these workers in the survey, the tech and humanitarian specialists drafted a funding application to launch the development of the

${ }^{14}$ The exact quote, in French, reads as follows: “L'art de la guerre ne sera pas le seul à être transformé par les algorithmes. Tout le système humanitaire va devoir muter, au sein d'organisations internationales ellesmêmes en plein bouleversements" (Nordmann 2018). 
system. Given their contributory expertise in research, the tech specialists took a prominent role in the formulation of the research grant application. This application was submitted in September 2017 to the Humanitarian Project Fund (HPF), a unit inside THO that funds research projects that are expected to significantly contribute to the organization's social mission. A committee comprising engineers, researchers, and physicians interested in humanitarianism reviews each application. A few weeks later, in the fall of 2017, the HPF granted the funds to begin the innovation processes.

\section{Phase 2: Tapping into Inflated Expectations}

Attracting investors is a critical step in innovation processes. Funding applications are discursive exercises that draw on collective expectations, with the overt goal to "raise attention and legitimise investment" (van Lente 2012, 773, italics in original). A strong funding application builds on collective expectations about what a technology could reasonably accomplish in the near future. The application filed for VirtEYE was no exception to this rule. In this application, collective expectations of AI played a key role in legitimizing VirtEYE. As one of the humanitarian specialists explained: "whatever feature we intended to include, no matter what the end goal was, stating that we would utilize AI gave us a certain credibility to develop VirtEYE" (Claude, online interview, 2019). According to this actor, the collective expectations of automation were so inflated at the time that the mere mention of $\mathrm{AI}$ in and of itself justified the attribution of funding.

Securing funding from the HPF was critically important for developing the project. As such, considerable thoughts and energy went into drafting the application. Since the tech specialists had more experience in writing funding proposals, they took a prominent role in this exercise. They wrote:

VirtEYE leverages artificial intelligence as a mode of assisting human agents and operators; AI-assistance can automate time-consuming tasks, aid in data collection and management, enhance user capacities and capabilities, and ensure THO emergency specialists focus on complex analysis and decision-making. With the further integration of comprehensive machine-learning processes, VirtEYE has the potential to improve emergency monitoring and response performance in the region and beyond. (Funding Application 2017)

This passage is interesting in many respects. First, it taps into the inflated collective expectations of AI at the time: the targeted readers could effortlessly associate the project with a technoscientific vision of automation that had received significantly positive media coverage. When optimistic collective expectations in a technology are so widely shared in society, the need to specify the technicalities of a project to develop this technology may seem unnecessary. For instance, when the tech specialists contended that "AI-assistance can automate time-consuming tasks," they took as their premise the widely shared belief that digital technologies will sooner or later take over manual tasks, without having to explain the minutia of how digital technologies would do so. 
Second, and related to the first, this excerpt bridges the gap between a general vision about automation-"AI will help a lot of people"-and its application in a specific context of humanitarianism- "AI will help humanitarian workers in their routine operations." The authors of the funding proposal achieved this connection by pointing to broad functions of AI and signaling them as evidently relevant to humanitarian actions. The text suggests that humanitarian workers will not be forgotten in the societal deployment of a techno-scientific vision of automation; they, too, will benefit from this new technology.

Third, the verb tenses and modifiers in this excerpt give both the impression that $\mathrm{AI}$ is already delivering results and that more extraordinary outcomes are forthcoming. The first sentence tells us that the project "leverages" AI, which is a technology that "can" do many great things. The second sentence points more explicitly toward the future, with "further integration" of the technology opening up a great "potential." Although no AI-powered component was operational in the humanitarian sector at the time that this funding application was submitted, the text induces a belief that the development of such components is inevitable.

Finally, the excerpt uses formulations to alleviate one apprehension that has always been an integral part of pessimistic techno-scientific visions about automation: the human fear of being replaced by intelligent machines. On this aspect, the techno-optimist literature on digital humanitarianism suggests that AI should be deployed to assist humanitarian workers rather than replace them (Meier 2015). The excerpt thus emphasizes the productive potential of AI, while refuting the primary apprehension around its deployment. The funding application conveys the message that the GIS will realize the techno-scientific vision of automation, and, as a result, VirtEYE will bring humanitarian practices on par with the cutting-edge possibilities of AI.

\section{Phase 3a: Disappointment I}

The HPF allocated funds for the VirtEYE project in the fall of 2017, but not without requesting clarifications as to how AI could be leveraged on a digital humanitarian platform. The VirtEYE team replied that the development of AI would take place in the last phase of project development. It listed two specific expectations about AI that were envisioned to significantly enhance humanitarian workflows: (a) a chatbot; and (b) a machine-learning technology that could be exploited to draw information from the institutional memory database. Once the funds were received, the team began to develop VirtEYE right away. The division of roles in the team followed the distribution of expertise: the tech specialists were tasked with programming the GIS, and the humanitarian specialists took a managerial role to ensure that VirtEYE would address the organizational needs of THO. 


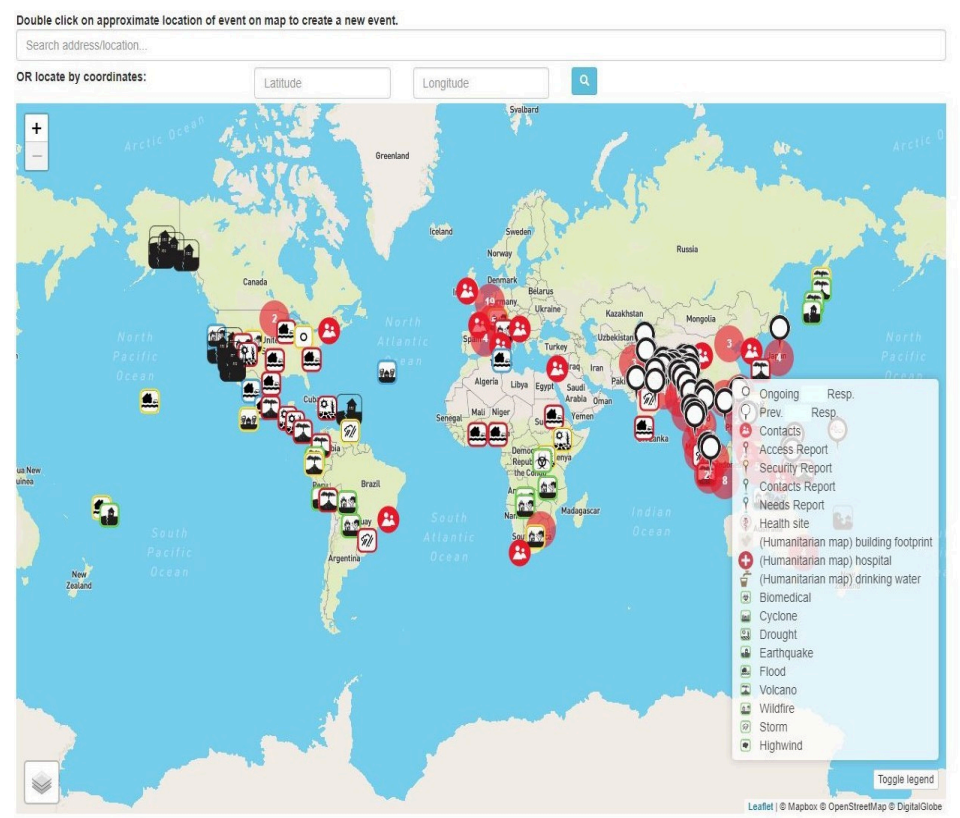

Figure 2: Snapshot of the landing page of VirtEye (2019 version)

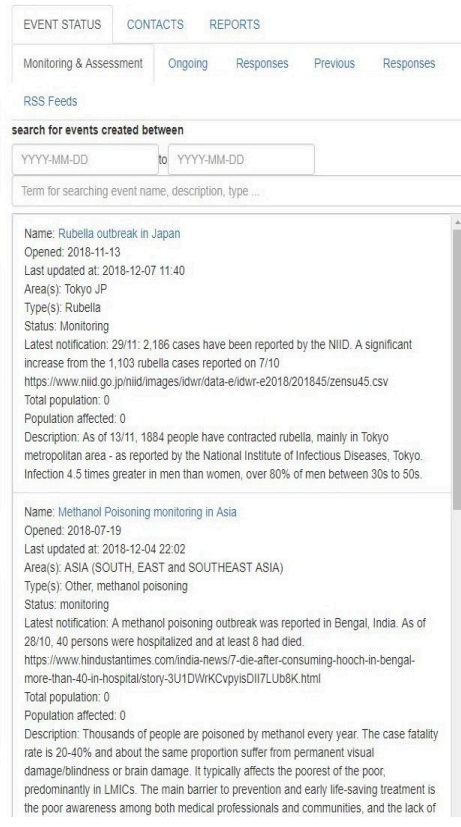

The general development of the system proceeded swiftly (see Figure 2). The GIS displayed a variety of sources of information, from automated RSS feeds to report cards updated manually by workers in the field. Together, these sources of information were displayed in the system to generate real-time and actionable forms of knowledge to enhance response efficiency in times of humanitarian crisis. However, the team quickly encountered problems in realizing the rather vague promises it had made pertaining to AI. The tech specialists' specific expectations did not match those of the humanitarian specialists. In their earlier project in Jakarta, the tech specialists had built a chatbot with rudimentary discursive capacities-one that automatically links delivered answers to specific keywords found in human requests. For VirtEYE, the tech specialists envisioned a much more powerful chatbot-one which would automate a human-like dialogue with THO contacts in the field. Alexis, a tech specialist who played a prominent role in the coding of VirtEYE, first tried to implement a machine-learning algorithm behind the chatbot, but they quickly realized that an AI-powered chatbot would be "too fragile" (pers. online interview, 2018). So the tech specialists reverted back to a simple keyword chatbot that would search through the database, like that chatbot deployed in the project deployed in Jakarta. While showing us the chatbot in a video conference, Alexis said:

It [the chatbot] takes the word that I type in and searches through the database. It is a bit of a fuzzy text search; so it will handle typos . . . if there is a partial match, it will return back whatever hit it will find. It's a simple text search, with a bit of fuzziness to it, and that's it. It's not really an AI/ML [machine learning], but the idea is that it looks a bit smart. (pers. interview, online interview, 2018) 
The realization of the first specific expectation, the AI-chatbot, proved to be more challenging than first anticipated. Therefore, drawing on their contributory expertise, the tech specialists used and improved an already-existing feature from their previous project in Jakarta to make VirtEYE into a platform that seemingly features "intelligence."

Yet, even the development of "a bit smart" chatbot eventually came to a halt. A smart chatbot was a more deliverable promise than an AI-powered chatbot, but it did not necessarily address the needs of the humanitarian specialists. In the project deployed in Jakarta, chatbots were designed to simulate a human-like dialogue with as many people as possible in order to collect information through social media and instant messaging. Such crowdsourcing of information from unknown sources through chatbots may be useful for collecting a large amount of data. However, a massive inflow of crowd-sourced data has downsides, too. In the context of VirtEYE, the humanitarian specialists feared that the deployment of a smart chatbot would increase the workload of VirtEYE operators, who would in turn have to parse through the collected information to select that which was actionable to display on the GIS. This illustrates how Collins and Evans' interactional expertise is important at the micro-level of the social dynamics of expectations: the tech specialists' perceptions of which AI applications would be useful for humanitarian interventions was not shared by the humanitarian experts. Based on their own contributory expertise, both groups of specialists had different specific expectations about how a smart chatbot could enhance humanitarian action.

The humanitarian specialists asked the tech specialists to abandon the development of the smart chatbot. Instead, the humanitarian specialists asked the tech specialists to focus on the implementation of a database of trusted sources of information, geo-localized on the map. Rather than passively collecting all sorts of information through an automated chatbot, the humanitarian specialists were more interested in creating access to a catalogue of local experts, all displayed on the GIS. VirtEYE users would be able to proactively target a trusted source of information in a given area, request an input of information from that source to be automatically uploaded to the system, and thus make more reliable data available on the GIS in a timely manner.

The success of VirtEYE hinges on its early adoption by humanitarian workers at THO, and as such, both the tech and humanitarian specialists had to concentrate on the development of features that could significantly improve the workflows of those humanitarian workers. In other words, if the project's takeoff was fueled by collective expectations of AI, its safe landing in institutional practices required the deployment of AI features that were of value to humanitarian workers. The aborted development of the smart chatbot illustrates this well. The tech specialists may have had the appropriate contributory expertise needed to develop a chatbot, but they lacked sufficient interactional expertise to devise an appropriate solution to the specific needs and expectations of the humanitarian specialists. 


\section{Phase 3b: Disappointment II}

If the humanitarian specialists dismissed the potential benefits of an AI-powered or "a bit smart" chatbot, they did not forget the second specific expectation promised in their funding application-an AI-powered functionality that can draw relevant information from institutional memory. Humanitarian specialist Claude describes their expectations regarding AI in an interview, two years later:

At the beginning, we were hoping to get an AI-based feature that would help information management. ... We wanted a feature that would, in a click of a button, give to users all the relevant information that they might need from our institutional memory. This was our dream. (online interview, 2019)

These actor-specific expectations were ultimately more difficult to realize than the smart chatbot. The tech specialists did not have the contributory expertise required to realize Claude's dream. For it to come true, the expertise of other specialists had to be sought. Since the tech specialists had greater technological understanding of AI than the humanitarian specialists, as well as an extended network of expert contacts in computer science, they took responsibility for finding a consulting firm to outsource the development of this second AI-powered feature.

The institutional memory on VirtEYE has been carefully structured. Since 2018, volunteers and freelancers have been uploading data from past THO missions on a server. Each entry has been linked to a geotagged event on the GIS. Any authorized user can thus access files from past events (e.g., daily reports, contacts, maps) through VirtEYE. Over time, the humanitarian specialists expected that VirtEYE users would create geo-localized event entries that would be stored on the server for future queries. Through this process, it was thought that the institutional memory of the organization would grow richer.

The consulting firm hired to realize the promise of AI-powered memory retrieval presented itself as an expert in AI solutions. The firm's primary task was to adapt its proprietary algorithms to find and extract information from the organization's institutional memory that was relevant to upcoming humanitarian crises-based specifically on the nature of the emergency and its location. This consulting firm brought contributory expertise in applied machine learning to the team, but the firm's staff did not have prior experience working in the humanitarian sector.

The contract was signed in May 2018. Between May and July 2018, the consulting firm worked on a copy of the database on its own server to develop the code that was intended to run in the background when users clicked a button labeled "Analyze." The specific expectation of the humanitarian specialists was that, with a single click, the proprietary algorithm would retrieve relevant and specific information from the organization's voluminous institutional memory (see Figure 4). In the months leading up to the delivery of the "Analyze" button, optimism about AI reached a peak. Claude recalled: "We were really enthusiastic. The experts were professionals. It seemed like they understood our needs." Claude explained: 
I had spent a lot of time to explain to them how we work as an organization [at THO]. It is important to do some "knowledge transfer" [about humanitarian workflows] to experts who assume that their technology will by itself revolutionize humanitarian actions. (Claude, online interview, 2018)

With only rudimentary interactional expertise in AI and computer science, Claude had heavily relied on the tech specialists' contributory expertise to code VirtEYE and the consulting firm's contributory expertise to develop the AI-powered "Analyze" button. However, Claude had actively participated in the innovation processes to ensure that the GIS would meet THO's organizational needs. Unlike in the chatbot, the humanitarian specialists saw in the "Analyze" button a real opportunity to feature VirtEYE as an innovative organizational solution to the perennial issue of efficient information management during humanitarian crises. The humanitarian specialists thus spared no effort to work toward the realization of their specific expectations.

\begin{tabular}{ll} 
Area(s) & $\begin{array}{l}\text { Papouasie-Nouvelle-Guinée high severity } \\
1 \mathrm{~km}\end{array}$ \\
& $\begin{array}{l}\text { East New Britain Province PG high severity } \\
3 \mathrm{~km}\end{array}$ \\
& monitoring \\
\hline Status & Earthquake \\
\hline Type & $2019-05-1416: 22$ \\
\hline Event Date and Time &
\end{tabular}

Figure 3: The "Analyze" button on the VirtEYE platform in early 2019.

Team members' expectations were still extremely optimistic when the consulting firm delivered the new AI-feature three months later. As Figure 3 illustrates, once clicked, the "Analyze" button launches a machine-learning algorithm that mines the database linked to VirtEYE. On the basis of findings from vicinal areas, the platform then suggests relevant information, files, maps, or contacts to the user. The humanitarian specialists tried the "Analyze" button several times, scrutinizing each result, before coming to the realization that the algorithm was not delivering on the expected promise. As Claude recalls, "we were receiving information relevant to Rwanda missions, while we were looking for information that pertains to missions in the Philippines." For Claude and the rest of the team of developers, the failings of the "Analyze" button dealt a blow to their optimistic expectations of AI.

For more than a year by that point, hype about AI had in large part legitimized the development of VirtEYE. However, after testing the "Analyze" button, the humanitarian specialists realized that the tool would not deliver the anticipated outcomes in its current form. The "Analyze" button could not be embedded in VirtEYE without additional work, including data cleaning and fine-tuning of the AI algorithm. To integrate AI into VirtEYE, the consulting 
firm would have to significantly improve the functionality; however, this expenditure had not been a priori budgeted for and could thwart the rest of the platform development. On the other hand, if the team of heterogeneous specialists did not deliver an AI-powered feature on VirtEYE, they would fail to deliver on their promise to funders. This predicament left the team with challenging decisions to make, as VirtEYE had less than a year of development left before its scheduled launch date.

\section{Phase 4: Recalibration of Expectations}

In the weeks that ensued, both the tech and humanitarian specialists presented the GIS to potential sponsors within THO in an effort to gain more VirtEYE adopters. Since the early stages of the project, the techno-scientific vision of automation in humanitarianism had taken a prominent place in the innovation processes of the GIS. Across THO, VirtEYE had become known as a project that would leverage AI to improve information management. As a result, in "live demo" presentations, THO workers expected to see the results of these innovation processes. "As soon as we had something, we tried to show it to [THO workers]," Claude explained. By leveraging collective expectations of AI as a generative force, live demos had previously provided an opportunity to showcase the vision of automation in humanitarianism, with the overt purpose of promoting wider adoption of VirtEYE at THO. However, the results produced by the "Analyze" button were so disappointing that the humanitarian specialists feared that the live demonstration of the AI would undermine what the rest of the GIS could actually accomplish. "We decided to change our approach. Instead of showing what the button "Analyze" could do, we presented a slide that showed the results that we would have wanted," Claude reported (see this slide in Figure 4).

In the summer of 2018, the tech and humanitarian specialists planned an additional eight months of development to implement, among other things, the AI-powered feature promised in the first funding application back in 2017. Hope had not vanished. There was still time to find and develop an alternative to the disappointing "Analyze" button.

The tech specialists justified the poor performance of the "Analyze" button as a temporary drawback in the implementation of an AI-powered feature on VirtEYE. Over time, they contended, as more events were created on the platform, the database linked to VirtEYE would become more voluminous. With more data, the proprietary algorithm could be expected to output better results. According to Alexis, it was just a matter of time before the "Analyze" button eventually generated actionable information (online interview, 2018). However, this statement about what AI could accomplish in the near future for humanitarian workers remained an expression of a specific expectation of $\mathrm{AI}$ in that context. As van Lente notes: "a project that fails now may promise to deliver something in the future" $(2012,774)$. 


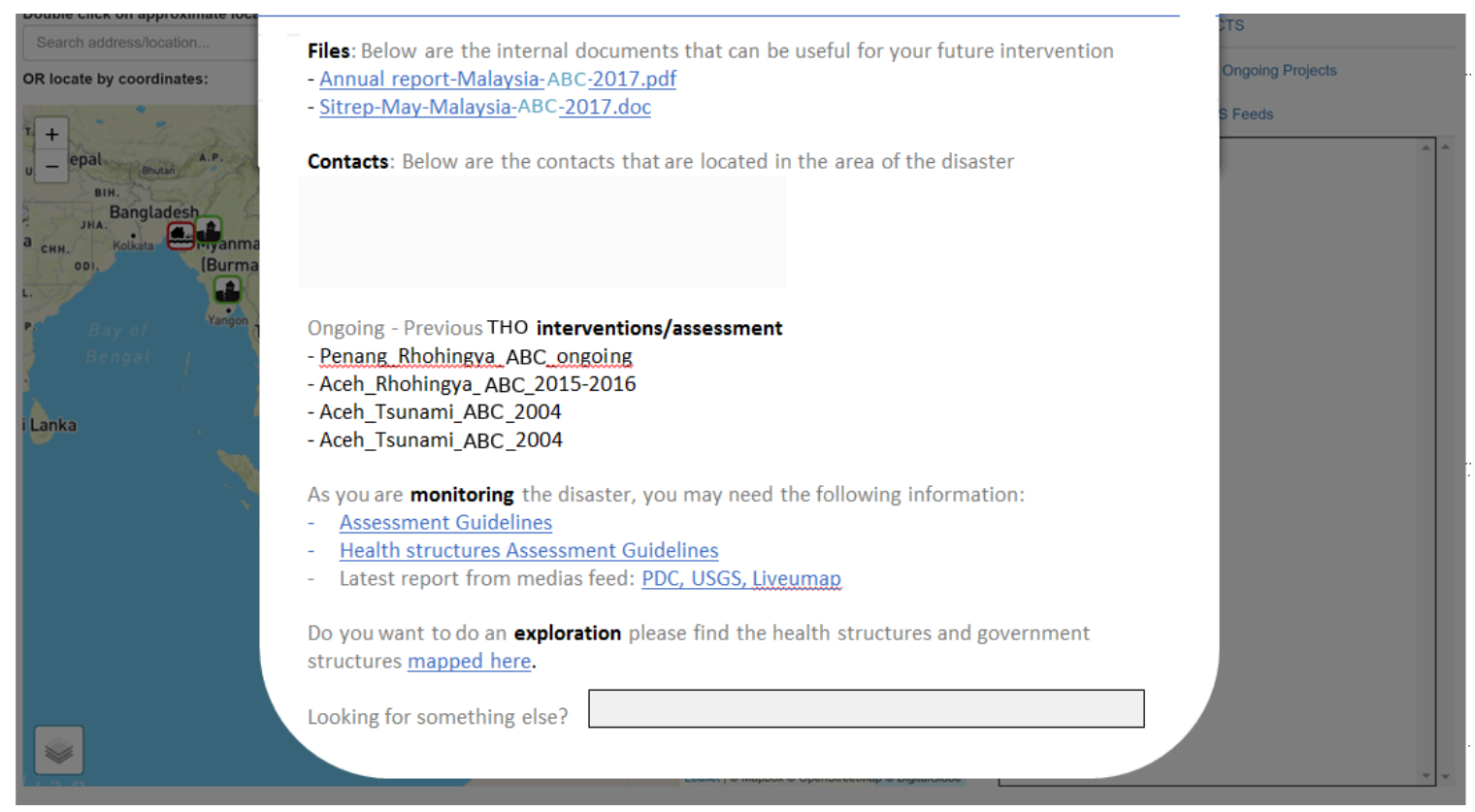

Figure 4: Claude's expected results for the AI-powered "Analyze" button.

In the early fall of 2018, the humanitarian specialists continued to explore ways that VirtEYE could use AI for information management. The disappointing performance of the "Analyze" button was compounded by the fact that it remained the intellectual property of the consulting firm. Given the limited resources available, the humanitarian specialists searched for an open-source (and free) alternative that could replace what had until then proven to be a disappointing AI-based feature. Later that fall, they established a new partnership with a small multidisciplinary team of social scientists-the authors of this article. The main objective of this collaboration was to "[r]esearch and report on how THO can better manage data/information in times of emergencies with technology such as AI (artificial intelligence) and ML (machine learning)" (contract between VirtEYE and the authors, September 2018). Based in part on our own research on $\mathrm{AI}$ and digital humanitarianism, we suggested that they reallocate VirtEYE's scarce resources to the development of features that would address the priority needs of future users. Contrary to the specific expectation that AI was bound to enhance information management in times of crisis, we argued that AI may "not necessarily be the best ... options to meet specific needs" of humanitarian workers and that "the specific needs that are best met by an option including AI are not necessarily the specific needs that the VirtEYE team should prioritize" (Claveau et al. 2018, 8). As the months passed, partially due to our own research output, the humanitarian specialists re-calibrated their specific expectations of AI, renounced the further development of any AI-powered feature resembling the "Analyze" button, and focused on the delivery of VirtEYE to early adopters who would test the platform. The promises of AIbased features may not have been delivered, but, according to Claude, VirtEYE remains an 
innovative and useful tool that did not previously exist at THO. Throughout these first two years of the innovation processes, collective expectations of the general usefulness of AI-associated with a highly prevalent and positive techno-scientific vision about automation-induced actorspecific expectations. The same collective expectations helped both the tech and humanitarian specialists to formulate explicit promises about how AI-powered automated features would significantly enhance humanitarian action. These promises helped to attract institutional support for the development of VirtEYE. These innovation processes highlight the critical significance of heterogeneous expertise in the interpretation of collective expectations that are produced out of the interaction of actors.

\section{Concluding Remarks}

In this article, we analyze a case study of the recent wave of enthusiasm for AI in humanitarian contexts. Our main presupposition was that, with respect to their social dynamics, expectations of AI are similar to expectations of any other technology. Like membrane technology (van Lente and Rip 1998), interactive TV and e-commerce (Konrad 2006), or biotechnology (Brown and Michael 2003), techno-scientific innovations of what is commonly referred to as AI follow general propositions that are well established in the sociology of expectations. Indeed, since the 1990s, the sociology of expectations has explored the impacts of real-time representations of future technological situations and capabilities on a wide array of technologies. We posit that AI is like any of these technologies. This premise led us to examine how social dynamics of expectations of AI shape innovation processes, just as these dynamics would do for any other technology.

In the theoretical section of this article, we examined six general propositions that stem from the sociology of expectations. In our case study, we used these propositions to scrutinize how expectations of AI in the humanitarian sector drive technological development. First, we probe how value-laden the dominant representations of a technological future were at the inception of the project. Imbued with hope, the expectations formulated in the project's funding application had a strong positive valence. Specifically, AI was expected to make humanitarianism more efficient. Second, the expectations at play ranged from a generic vision about the benefits of automation to the very narrow prediction that an AI-powered "Analyze" button could solve an important information need of THO workers. Third, the strength of expectations conformed to a typical temporal pattern, with initial enthusiasm followed by disappointment and re-calibration. Fourth, expectations clearly generated something: the actors involved launched the project because they were enthusiastic about the potential of AI in the humanitarian sector; they were funded and supported by THO because they effectively tapped into the hype about this technology; and they persevered in the face of disappointing preliminary results because they believed the technology could be beneficial. Fifth, actors involved with VirtEYE formulated their own expectations-what we call actor-specific expectations-from collective expectations that are not attributable to specific individuals or group of individuals. In this case study, actor-specific 
expectations initially aligned with dominant collective expectations. ${ }^{15}$ However, as innovation processes unfolded, individual actors came to formulate their own specific expectations of how AI could be applied in the humanitarian sector. Finally, the tech and humanitarian specialists on the team shared similar optimistic visions of AI in the humanitarian domain, but their narrow, actor-specific expectations were heterogeneous. Beneath their seemingly congruent optimistic visions of AI lay significant gaps in how the actors negotiated their own specific expectations at the micro-level. This is where this paper brings a contribution to the sociology of expectations. We contend that the "Studies on Expertise and Experience" literature is helpful for grasping those particular, micro-level dynamics of innovation processes.

The main theoretical contribution of this article consists in how we applied the insights of Collins and Evans $(2002,2007)$ on experience and expertise to better capture the intricacies of the social dynamics of expectations at the micro-level. We argue that the distribution of expertise that is leveraged in the context of technological development shapes both the formulation of actorspecific expectations and the interactions of individuals involved in innovation processes. Our analysis illustrates how Collins and Evans' typology of contributory and interactional expertise helps to increase understanding of the heterogeneity and interactions of actors during innovation processes. Actors translate generic collective expectations into narrower actor-specific expectations on the basis of their contributory expertises. The expectations of actors with different specialist expertises will thus tend to have different contents. In turn, the degree of interactional expertise that a specialist has enables them to be more fluent in the expertises of other specialists. Such interactional expertise lends credence to a synergetic partnership.

In the context of VirtEYE, each group of specialists had specific understandings of how best to materialize collective expectations of AI for the humanitarian sector. Building on a prior project, the tech specialists saw promise in an AI-powered chatbot. In contrast, the humanitarian specialists sought an AI-powered "Analyze" button, in part because of their deep knowledge of humanitarian practices and their need for a quick way to leverage actionable knowledge from the institutional memory of the organization. Both tech and humanitarian specialists constructed their own specific expectations on the basis of their own set of competencies and experiences. However, while both possessed fluency in their respective domains of contributory expertise, both also had shortcomings in their interactional expertise. The tech specialists failed to quickly identify how to best leverage the capability of AI for THO workflows. As for the humanitarian specialists, their limitations in grasping the mechanics of machine learning led them to be overenthusiastic about what AI could realistically accomplish for VirtEYE users.

Actor-specific expectations are mutable, and interactions with other actors transform them. However, a convergence or even reciprocal understanding of expectations in a group of heterogeneous specialists is not bound to happen without some degree of interactional expertise.

${ }_{15}$ Since our research focused on a group of actors that are not central to the social construction of collective expectations of AI, we have not studied the other direction of influence passing through discourse and innovation activities, from actor-specific expectations to collective expectations (see Figure 1). We conjecture that as time goes by, the multiplication of micro-level failed attempts at realizing optimistic expectations of a technology explains the deflation of hype. 
As Collins notes, "mutual understanding and coordination comes through interactional expertise" (2018, 74). Our examination of the successive phases of VirtEYE development exemplifies this. Like any other technology under development, promissory expectations of AI act on innovation practices and processes. As the social dynamics of expectations evolve, individual interpretations of collective expectations become plural and diverse. Inevitably, the coalescence of heterogeneous expertises involved in technological development leads to the negotiation of innovation processes. While the sociology of expectation teaches us that actorspecific expectations emerge from these social interactions, our case study suggests that not only are heterogeneous contributory expertises central to the social dynamics of expectations, but so too are interactional expertises.

\section{Author Biography}

At the time of writing, Guillaume Dandurand was Postdoctoral Fellow in the Département de philosophie et d'éthique appliquée, Université de Sherbrooke. Guillaume is now Postdoctoral Fellow in the Institut national de la recherche scientifique. In 2019, Guillaume was awarded the Dissertation Prize by York University for his doctoral research on the digitization of welfare in India.

\section{Author Biography}

François Claveau is Associate Professor in the Département de philosophie et d'éthique appliquée, Université de Sherbrooke. François holds the Canada Research Chair in Applied Epistemology, is a regular member of the Centre interdisciplinaire de recherche sur la science et la technologie (CIRST) and of the Centre de recherche en éthique (CRÉ). He has recently been awarded the Prix Tremplin from Université de Sherbrooke for his original contribution to research on digital humanities.

\section{Author Biography}

Jean-François Dubé teaches philosophy at the Cégep de Granby and is a former humanitarian worker. Jean-François has built a unique research portfolio in action research, applied epistemology, and digital humanitarianism. Jean-François is also a member of the Observatoire international sur les impacts sociétaux de l'IA et du numérique.

\section{Author Biography}

Florence Millerand is Professor in the Département de communication sociale et publique, Université du Québec à Montréal. Florence also co-supervises the Laboratory for Communication 
and the Digital and is a regular member of the Centre interdisciplinaire de recherche sur la science et la technologie.

\section{Acknowledgements}

We are grateful to all of the interlocutors who helped us better understand innovation processes in the humanitarian context. This paper greatly benefited from valuable exchanges with Claude, Guillaume Gagnon, Sébastien Gambs, Florence Lussier-Lejeune, Dominic Martin, Marie-Jean Meurs, the research community at the Centre interdisciplinaire de recherche sur la science et la technologie, and the participants of the 2019 Summer Research Retreat organized by the Canada Research Chair in Applied Epistemology. We would also like to extend our gratitude to Katie Vann and to the anonymous reviewer for their insightful comments. This article has received financial support from the Humanitarian Project Fund, Mitacs [IT11305], the Centre interdisciplinaire de recherche sur la science et la technologie, the Social Sciences and Humanities Research Council [892-2019-1057], and Canada Research Chairs [950-230644].

\section{References}

Borowiec, Steven. 2016. "AlphaGo Seals 4-1 Victory over Go Grandmaster Lee Sedol." The Guardian, March 15. https:// www.theguardian.com/technology/2016/mar/15/googlesalphago-seals-4-1-victory-over-grandmaster-lee-sedol.

Borup, Mads, Nik Brown, Kornelia Konrad, and Harro van Lente. 2006. "The Sociology of Expectations in Science and Technology." Technology Analysis \& Strategic Management 18(3-4):285-98. doi:10.1080/09537320600777002.

Brown, Nik, and Mike Michael. 2003. "A Sociology of Expectations: Retrospecting Prospects and Prospecting Retrospects." Technology Analysis \& Strategic Management 15(1):3-18. doi:10.1080/0953732032000046024.

Burns, Ryan. 2014. "Moments of Closure in the Knowledge Politics of Digital Humanitarianism." Geoforum 53(complete):51-62. doi:10.1016/j.geoforum.2014.02.002.

—_ 2015. "Rethinking Big Data in Digital Humanitarianism: Practices, Epistemologies, and Social Relations." GeoJournal 80(4):477-90. doi:10.1007/ s10708-014-9599-x.

Claveau, François, Guillaume Dandurand, Jean-François Dubé, Florence Millerand. 2018. Situating VirtEYE: First Progress Report. Montréal: Centre interdisciplinaire de recherche sur la science et la technologie.

Collins, Harry. 2018. “Studies of Expertise and Experience." Topoi 37(1):67-77. doi:10.1007/s11245016-9412-1.

Collins, Harry M., and Robert Evans. 2002. "The Third Wave of Science Studies. Studies of Expertise and Experience." Social Studies of Science 32:235-96. doi: $10.1177 / 0306312702032002003$.

2007. Rethinking Expertise. Chicago: University of Chicago Press. 
Crawford, Kate, and Megan Finn. 2015. "The Limits of Crisis Data: Analytical and Ethical Challenges of Using Social and Mobile Data to Understand Disasters." GeoJournal 80 (4): 491-502. https: / / doi.org/10.1007/s10708-014-9597-z.

Dedehayir, Ozgur, and Martin Steinert. 2016. "The Hype Cycle Model: A Review and Future Directions." Technological Forecasting and Social Change 108(July):28-41. doi:10.1016/j.techfore.2016.04.005.

Duffield, Mark. 2016. "The Resilience of the Ruins: Towards a Critique of Digital Humanitarianism." Resilience 44 (3): https: / / doi.org/ 10.1080/21693293.2016.1153772.

Fenn, Jackie, and Mark Raskino. 2008. Mastering the Hype Cycle: How to Choose the Right Innovation at the Right Time. Boston: Harvard Business Review Press.

Ferrucci, David, Anthony Levas, Sugato Bagchi, David Gondek, and Erik T. Mueller. 2013. “Watson: Beyond Jeopardy!" Artificial Intelligence 199-200(June):93-105. doi:10.1016/j.artint.2012.06.009.

Geels, Frank W., and Wim A. Smit. 2000. “Lessons from Failed Technology Futures: Potholes in the Road to the Future." In Contested Futures: A Sociology of Prospective Techno-Science, edited by Nik Brown, Brian Rappert, and Andrew Webster, 129-55. London: Routledge.

Godin, Benoit, and Dominique Vinck. 2017. Critical Studies of Innovation: Alternative Approaches to the Pro-Innovation Bias. Cheltenham, UK: Edward Elgar Publishing.

Hassler, Susan. 2016. “2017: The Year Of Self-Driving Cars And Trucks.” IEEE Spectrum, December 30. https://spectrum.ieee.org/transportation/advanced-cars/2017-the-yearof-selfdriving-cars-and-trucks.

Holderness, Tomas, and Etienne Turpin. 2015. "PetaJakarta.Org: Assessing the Role of Social Media for Civic Co-Management during Monsoon Flooding in Jakarta, Indonesia." SMART Infrastructure Facility, University of Wollongong. https: / / uowvivo.uow.edu.au/display/publication109685

Jasanoff, Sheila, and Sang-Hyun Kim. 2009. "Containing the Atom: Sociotechnical Imaginaries and Nuclear Power in the United States and South Korea." Minerva 47(2):119. doi:10.1007/ s11024-009-9124-4.

Joly, Pierre-Benoit. 2015. “Le Régime Des Promesses Technoscientifiques.” In Pourquoi Tant de Promesses, edited by M. Audétat, 31-48. Paris: Hermann.

Konrad, Kornelia. 2006. "The Social Dynamics of Expectations: The Interaction of Collective and Actor-Specific Expectations on Electronic Commerce and Interactive Television." Technology Analysis \& Strategic Management 18(3-4): 429-44. doi:10.1080/09537320600777192.

Konrad, Kornelia, Harro van Lente, Christopher Groves, and Cynthia Selin. 2017. "Performing and Governing the Future in Science and Technology." In The Handbook of Science and Technology Studies, edited by Ulrike Felt, Ray Fouché, Clark A. Miller, and Laurel SmithDoerr, 4th ed., 465-94. Cambridge: MIT Press. 
Mark, David M., Nicholas Chrisman, Andrew U. Frank, Patrick H. McHaffie, and John Pickles. 1997. "The GIS History Project." National Center for Geographic Information and Analysis. Accessed 29 April 2020. http:/ / www.ncgia.buffalo.edu/ncgia/gishist/bar_harbor.html.

Meier, Patrick. 2012. "Crisis Mapping in Action: How Open Source Software and Global Volunteer Networks Are Changing the World, One Map at a Time." Journal of Map \& Geography Libraries 8(2):89-100. doi:10.1080/15420353.2012.663739.

—_ 2015. Digital Humanitarians: How Big Data Is Changing the Face of Humanitarian Response. Boca Raton, FL: CRC Press, Taylor \& Francis Group.

Mullany, Michael. 2016. "8 Lessons from 20 Years of Hype Cycles." Pulse, December 7. Accessed 29 April 2020. https://www.linkedin.com/pulse/8-lessons-from-20-years-hype-cyclesmichael-mullany.

Mulkay, Michael. 1993. "Rhetorics of Hope and Fear in the Great Embryo Debate." Social Studies of Science 23 (4): 721-42.

Nordmann, François. 2018. “Comment Fonctionnera l'humanitaire En 2030?" Le Temps, December 3. https: / / www.letemps.ch / opinions / fonctionnera-lhumanitaire-2030.

Roth, Silke, and Markus Luczak-Roesch. 2020. "Deconstructing the Data Life-Cycle in Digital Humanitarianism." Information, Communication $\mathcal{E}$ Society 23 (4): 555-71. https: / / doi.org/10.1080/1369118X.2018.1521457.

Schwab, Klaus. 2017. The Fourth Industrial Revolution. New York: Crown Business.

Stilgoe, Jack. 2018. "Machine Learning, Social Learning and the Governance of Self-Driving Cars." Social Studies of Science 48(1):25-56. doi:10.1177/ 0306312717741687.

van Lente, Harro. 1993. "Promising Technology: The Dynamics of Expectations in Technological Developments." PhD diss., Universiteit Twente.

van Lente, Harro. 2012. "Navigating Foresight in a Sea of Expectations: Lessons from the Sociology of Expectations." Technology Analysis \& Strategic Management 24(8):769-82. doi:10.1080/09537325.2012.715478.

van Lente, Harro, and Arie Rip. 1998. "Expectations in technological developments. Prospective structures to be filled in by agency." In Getting New Technologies Together: Studies in Making Sociotechnical Order, edited by Disco Cornelis and Barend van der Meulen, 203-31. New York: Walter de Gruyter. https:// research.utwente.nl/en/publications/expectations-intechnological-developments-prospective-structures.

van Lente, Harro, Charlotte Spitters, and Alexander Peine. 2013. "Comparing Technological Hype Cycles: Towards a Theory." Technological Forecasting and Social Change 80(8):1615-28. doi:10.1016/j.techfore.2012.12.004.

Vertesi, Janet, David Ribes, Laura Forlano, and Yanni Loukissas, Marisa Leavitt Cohn. 2017. "Engaging, Designing, and Making Digital Systems." In The Handbook of Science and Technology Studies, edited by Ulrike Felt, Ray Fouché, Clark A. Miller, and Laurel SmithDoerr, 4th ed., 169-93. Cambridge: MIT Press.

Vinatier, Isabelle, and Joëlle Morissette. 2015. "Les Recherches Collaboratives: Enjeux et Perspectives." Carrefours de l'éducation 39(1):137-70.

Yin, Robert K. 2003. Case Study Research: Design and Methods. London: Sage. 